Results No evidence of detrimental effects of maternal employment in the early years on subsequent child socio-emotional behaviour was seen. There were significant gender differences in the effects of parental work arrangements on behavioural outcomes. Girls whose mothers were not in paid work during their first 5 years were $77 \%$ (95\% CI 1.21 to 2.57 ) more likely to have behavioural difficulties at age 5 than girls whose mothers were in paid work throughout their early years, independent of maternal characteristics and household income. For boys this was not the case, but boys in two-parent households in which their father was not in paid work for at least one period during their first five years were at an increased risk for behavioural problems at age 5 . The most beneficial working arrangement for both girls and boys was that in which both mothers and fathers were present in the household and in paid work, independent of parental educational attainment and household income.

\section{IT IS NOT "JUST DEPRIVATION": WHY DO EQUALLY DEPRIVED UK CITIES EXPERIENCE DIFFERENT HEALTH OUTCOMES?}

doi:10.1136/jech.2010.120956.83

${ }^{1} \mathrm{D}$ Walsh, ${ }^{2} \mathrm{~N}$ Bendel, ${ }^{3} \mathrm{R}$ Jones, ${ }^{4} \mathrm{P}$ Hanlon. ${ }^{1}$ Glasgow Centre for Population Health, Glasgow, UK; ${ }^{2}$ NHS Manchester/Manchester Joint Health Unit, Manchester, UK; ${ }^{3}$ Liverpool Primary Care Trust, Liverpool, UK; ${ }^{4}$ University of Glasgow, Glasgow, UK

Background The link between socio-economic circumstances and health is well established, with material deprivation cited by UK politicians and policy-makers as the principal underlying cause of poor health and low life expectancy. However, research has shown that not all poor health can be explained purely in terms of deprivation: Glasgow in particular has been cited in this regard, with a "Glasgow effect" suggested to explain the city's high mortality rates. However, Glasgow is not alone in experiencing relatively high levels of poor health and deprivation within the UK: Liverpool and
Manchester are very similar in this regard. Previous analyses of this "effect" were constrained by limitations of data and geography.

Objectives To establish whether there is evidence of a so-called Glasgow effect: (a) even when compared to its two most similar and comparable UK cities; and (b) when based on a more robust and spatially sensitive measure of deprivation than was previously available to researchers.

Participants and setting Total populations of Glasgow, Liverpool and Manchester.

Design Rates of "income deprivation" (a measure very highly correlated with the main UK indices of multiple deprivation) were calculated for small areas in the three cities. All-cause and causespecific SMRs were calculated for Glasgow relative to Liverpool and Manchester, standardising for age, sex and deprivation decile. In addition, a range of historical census and mortality data were analysed.

Results The deprivation profiles of Glasgow, Liverpool and Manchester are almost identical. Despite this, premature deaths in Glasgow are more than 30\% higher, with all deaths around 15\% higher. This "excess" mortality is seen across virtually the whole population: most age groups, both males and females, in deprived and non-deprived neighbourhoods. For $<65 \mathrm{~s}$, SMRs tended to be higher for the more deprived areas, and around a half of "excess" premature deaths were directly related to alcohol and drugs. Analyses of historical data suggest that the deprivation profile of Glasgow has not changed significantly relative to Liverpool and Manchester in recent decades; however, the mortality gap appears to have widened since the early 1970 s, suggesting that the "effect" is a relatively recent phenomenon.

Conclusion While deprivation is a fundamental determinant of health and, therefore, an important driver of mortality, it is, however, only one part of a complex picture. As currently measured, deprivation does not explain the higher levels of mortality experienced by Glasgow in relation to two very similar UK cities. Additional explanations are required. 